\title{
PRECISION MULTI-DEGREES-OF-FREEDOM POSITIONING SYSTEMS
}

\author{
Modular Design For Assembly Applications
}

${ }^{1}$ Gheorghe Olea, 'Kiyoshi Takamasu and ${ }^{2}$ Benoit Raucent

${ }^{1 *}$ The University of Tokyo, 7-3-1 Hongo, Bunkyo-ku, 113-8656 Tokyo, Japan; ${ }^{2}$ Université Catholique de Louvain, Place de levant 2, B-1349 Louvain-la-Neuve, Belgium

Abstract: The paper is dealing with the development of a new class of multipurpose multi-degrees-o-freedom (MDOF) Positioning Systems (PS) to be used as precision devices in a cell manufacturing desktop environment. Based on a structural investigation and systematization with imposed criteria, PM with 2 dof actuators on the base was proposed. In its first modular design concept, each of the members-Positioning units( $\mathrm{Pu})$, having at least 2DOF motion capabilities over a large workspaces, are symmetric parallel mechanisms (PM) architectures, fully actuated, by in-parallel linear-linear (L-L) actuation modules $(\mathrm{aM})$. The kinematics and design aspects for the representative cases 2, 4 and 6 DOF $\mathrm{Pu}$, by using planar motors and scissors (and/or, parallelograms) as transfer of motion modules(Tof $M$ ) are presented, as they exhibit, both, an increased stiffness and speed features, respectively.

Key words: parallel mechanisms; positioning, desktop assembly; precision; multi-degreesof-freedom; 2dof actuators; modular design; parallelograms; planar motors.

\section{INTRODUCTION}

In the positioning technology $y^{1}$, for example, a new system, should exhibit not only an increased level of accuracy (as the main index of the performance), but some times, a higher speed and/or bigger payload capacity over a large work spaces. They must, generally, to be able to perform complex tasks motions with multi degrees of freedoms capabilities. A compact, small size is always welcomed (if, possible) and the modular

\footnotetext{
* Now working with Université Catholique de Louvain as Guest Researcher
} 
aspects taken in to account, for the flexibility of production. A development of such advanced systems to cope with all (or, some) of these requirements are to be (strong) innovative, including the latest theoretical results from the specific field(s), and the actual practical progresses in the technology domain (ex., linear direct drive technology, piezo,..).

This is also a case, with a positioning/manipulation system (P/MS) inside of a assembly cell from the semiconductor, optics, or more generally, precision manufacturing industry. Today, the assembly processes are still preponderant at the low level of automation, comparing with machining, for example. And, a lot of work is now focused on the manipulation at the micro/nano level for very small parts. But, there are still a lot of work to do, and gaps at the desktop level: desktop factory, automation cell, etc. Excepting simple task (transfer) which requires one DOF, most of the tasks here require more than two DOF( $>2 \mathrm{DOF})$ motion capability. They are to be (highly) precise $(<100 \mu \mathrm{m}$ ), fast enough (at least $1 \mathrm{~m} / \mathrm{s}$ ) and in a flexible manner (modules) done on a working table $(1000 \mathrm{~mm})$.

As a Positioning System (PS) always play an important role in an ensemble of a machine, equipment, device, etc. the choice of an adequate mechanical structure for a Positioning System (PS) will be the first step. The main concept in the design of standard multi degrees of freedom (MDOF) positioning systems, previously used for many years, have been based on the serial chain structures, when individual units (stages), with standard number of DOF (1) in translation or rotation, having a variable low accuracy (generally, ball screws) were chosen to be added by stacking one after another, to fulfill with the required total (multi)number of DOF, Motorized(robotic) axes on belt, rack/pinion or air transmission power systems have a good speed $(>1 \mathrm{~m} / \mathrm{s})$ and large strokes $(>500 \mathrm{~mm})$ but, are at the limit of the accuracy required in a precision assembly $(>0.1 \mathrm{~mm})$. With an appreciable load, the positioning tasks become difficult, increasing their drawbacks. Besides of the advantages of these two concepts - low cost and feasibility, and their high standardization in modules, they can not overcome with their drawbacks because of the vicious circle accuracy-speed-forcestiffness, characteristic of the in-serried structures (presented elsewhere) and their consequences (low stiffness, and poor dynamics behavior as the vibrations is increasing). In such case, the design concept on serial structures based cannot be seen as a solutions, and the PS not properly work.

To accomplish with these, parallel structures - have to come and solve the problem as they are appearing to be a good alternative to solve some of the insurmountable problems presented before. As a result PMs have been studied and researched intensively, in the last period of time, in this context and every time an increased accuracy, stiffness, and dynamics, were main requirements. Unfortunately, they have small workspaces and complicated 
kinematics/dynamics as the DOF increase. On the other hand, intelligent machines (like, SCARA robots) using the transfer line ${ }^{2}$ and/or planar motors ${ }^{3}$ as auxiliary devices systems in cooperation with other axes, prove to be a productive and adequate solution for manufacturing (assembly) in mass production of products. However, if the type and volume of the products is changing, less automated (robotized) system increased more in attention by their possibilities to be highly productive, in using their flexibility. Equipped with local and global intelligent sensors (like, CCD video camera) the new environment together with the "assistants" can manage various tasks inside. Especially, when workers are with different abilities and/or are subjected to constraints due the physical conditions this system should exhibits the usefulness. These assistants could be small robots working on a table or other new adequate systems with manipulation/positioning capabilities ${ }^{4}$.

In our paper an investigative work was conducted on the PMs general class with imposed design conditions (constrains) from above, in a systematic manner, in order to find a suitable solution to the previous problems. The study has had as a consequence the selection of PMs symmetric structures class, fully actuated which is using 2 dof actuators on the base, to be further developed, using the planar: a) in-parallel kinematics 2 dof actuators and b) simple and/or double scissors/ parallelogram transfer of motion mechanisms. With these improvements and others in the design solutions, or practical implementations, some of the members exhibit new and/or improved features. Their general kinematics and (pre)design considerations are presented as a single modular concept.

\section{PRECISION MULTI-DEGREES-OF-FREEDOM POSITIONING SYSTEMS (P/MDOF-PS)}

\subsection{Conceptual Design}

The conceptual design stage is the most important one, in any process of creation. New innovative products are based on this, and it is the crucial one. The final conclusions in the short (investigative) analysis from the introductory part were: a) PM structures could be a good (and, single alternative) solution to the serial structure design concept for PS, and b) The linear direct-drive technology is able and capable today to deliver advanced practical solutions for a variety of purposes (including, the planar one).

We adopted them as fundamentals principles of the conceptual design process. Then, with more deeply investigative actions, some systematization, and based on our own work and ideas, to be able to deliver the right solution at our design. We did it, and now shortly we are presenting the results. 


\section{Parallel Mechanisms as Positioning Systems(short investigation)}

PMs were from the beginning studied as mechanical systems to be used in manipulation, involving the positioning ones. In the recent years, an appreciable effort was done towards their investigation and development to be potential used in industry, based on the previous successful works and results. But, in the assembly, famous implementations still have to wait.

The first design as a manipulator has been done by MacCallion ${ }^{6}$ even for an assembly station. It has $6 \mathrm{DOF}$, three points actuated and used three pantographs to perform the insertion tasks. After that, some other researchers dealt with this aspect ${ }^{7,8}$, too. The PM as a compliant mechanism was studied at INRIA ${ }^{5}$, and one of the prototype shown their high capability to accurate sense an external force by accurate mapping the force. But, a first commercial parallel manipulator (GADFLY) was sold in assembly(ICintegrated circuit) by Marconi, only in 1992. Apart of the well-known yet structure, Gough-Stewart platform ${ }^{9}$, an enormous amount of new structures and design improvements are now on the "market" and were (or, are to be) proved as practical PS solutions. Their number is increasing dramatically, being difficult to be counted, and a data base maintained (and, updated). Occasionally, groups or individuals belonging to famous research centers or laboratories in the frame of small or large projects (ROBOTOOL, ..etc) tried and made good efforts to investigate as much as possible structures for some of their purposes (scientific, didactic, etc). An individual, but with constant and rigorous selection updates on INTERNET was doing by Merlet and the results published ${ }^{5}$ in a book which stand as a design guide. Most of the PMs structures have generally, only one(1) dof actuators, and with all the fixed points at the base. (Gough-Stewart PM in its general well-known design includes six linear actuators with all the $\operatorname{six}(6)$ points from one end, fixed on the base). In a normal (standard) design concept-motors, ball screws, open loop control, etc it not reached an impressive level of accuracy, and the small workspace is a result of a maximum number of joints used (6) and their complexity. Taken in to account the errors from this complicated actuation technology (guides, wear,..) the control becomes difficult. For tasks involving less than all six DOF, they are not well suited structures for manipulation and the coefficient WS/foot print is disadvantageous. Even if, PM seems to be highly desired in the industry, as micro/nano manipulation system (semiconductor, assembly,..), because of the specific features (high accuracy, small space, good stiffness,..) in our case of requirements (table top motion systems) the G-S PM will be not the first solution. Another wellknown PM famous structures, the DELTA ${ }^{10}$ and HEXA has as principle of actuation initially, the fixed points, but latter, depending on the applications these were moved in one direction (ex., LINAPOD,..) or, others(ex. TOYODA,..) by using the linear (ball screw, or, linear motor technology). 
One of the first solutions of PM with all unfixed points of the actuation, with a motion parallel with the base was done by Han and Tahmaseb $\mathrm{i}^{11}$, using four and five (pantograph) bar mechanisms, respectively, for each of their six actuators. By these solutions, and with fixed lengths links, the ws of the actuators in a plane was increased, but total WS not significantly. (Note, the actuators have a closed loop linkage, and in-parallel actuated structure, respectively.)

As it was stated in the claims ${ }^{11}$, the 6DOF parallel (mini)manipulator with 2 dof actuation concept, for fine position and force control in a hybrid serial-parallel system and with fixed lengths (limbs) was designed to provide high accuracy and stiffness, but it was not designed to provide very large displacements. Latter a possible combination of passive (SR) and actuation (stepper motor X-Y stages) joints was proposed.

$P M$ with 2 dof was used in two fundamental prototypes, by $\operatorname{Kohli}(\mathrm{R}-\mathrm{L})^{12}$ and Behi $(\mathrm{L} / \mathrm{L})$ disclosing the advantages derived from a symmetrical simplified structure. (Note, the first one gave a practical solution for inparallel actuation actuators, when the last was serial one.) First experimental implementation using planar motors was done with a kinematics different from previous ones by using S-R joints in one chain ${ }^{13}$. From then, other developments in this direction was done, too.

The work presented in this paper here is in somewhat an extension of what was partially done before. A special class of spatial symmetrically PM structures with 2dof actuators (R-L and L-L) and 6DOF capabilities, labeled as-type "A" was studied ${ }^{14}$ towards the development of new and innovative mechanisms with an improved potential and capabilities as positioning, carry load, speed,... no necessary all together. Two of them, in horizontal and vertical linear actuation: 6-3[(PP)RS]-H and $-\mathrm{V}$, respectively were from the positioning point of view analyzed, because of its appealing potential to be implemented as mechanisms for precision manipulations, by using (then) the new direct-drive linear technology. The actuated joints were conceived to work in three limited rectangular planes. As a result, the PM exhibited a workspace, insides only of the triangular base. Other technical solutions, by using the planar actuations, were proposed from the design pov, and for an immediate implementation, by using standard Japanese mechatronic products, components and technologies ${ }^{15}$ latter. Finally, a Parallel Positioning Device ${ }^{16}$ was developed.

Structural Systematization and Developments. Using the traditional tool of investigation applied on the actual PM data base (references) of structures, and based on our own experience and work, a process of systematization and development was conducted. The results are shown below, and will be discussed now shortly. 
Even from the beginning, we imposed several criteria to be followed in this systematization:

a) actuators should be on the base (from many good considerations, but mainly from the static and dynamics pov-stiffness, speed, force,...),

b) to use as much as possible the similar components (links and pair, opening the way towards the modularization, and flexibility of their manufacturing and assembly), and

c) few parts in motion (for dynamics and precision, diminishing the chance to difficult manage the manufacturing errors and/or the calibration).

As a first result (not shown here, for the space reason), we find that a closed loop PM structures with MDOF capability (2-6 DOF) and with 2dof actuation (from the level I and/or II), symmetrical, which have a reduced number of elements-links and pairs, (compared with that using 1 dof actuators) could be the first solution. The number of closed loops(branches) were the minimum for this PM class $-1(2)-5(6)$ but, from the entire class, finally we selected a subclass which shown the minimum number of components on a branch. For these, the actuators (all) are located only at one level (I), and should have incorporated at least two degree-of-freedom.
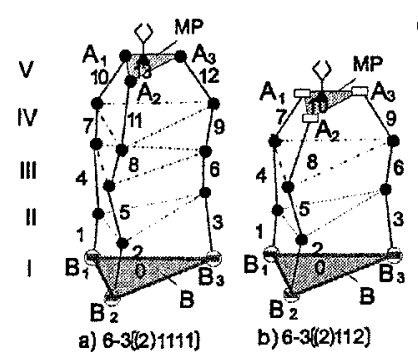

6 DOF

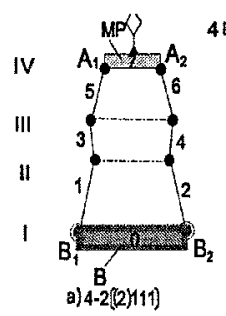

4 DOF
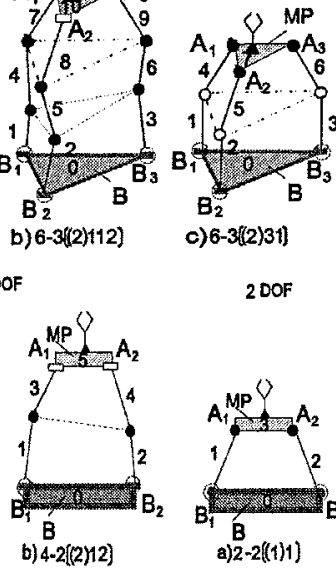

c)6-3(2)31)

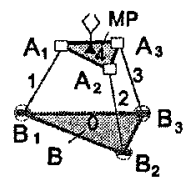

d) 6-3(2)4]

2 DOF

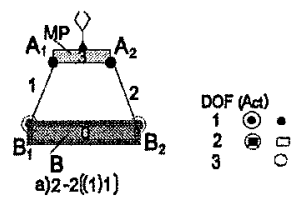

Figure 1.2dof actuated PM class(fundamental structures)-6, 4 and 2DOF (actuation)

This class we selected to be analyzed and discussed, and further developed. It has as components, two families - PMs with 4 and 6 DOFs, Fig.1. Several representative members as a standard combination of the pairs with $1,2,3$ and $4 \mathrm{dof}$ between levels(II-V) are shown and all the possible combination and their specific features synthesized and included in Tab.1. The structures 
belong to the symmetrical ones, general class. We should point out, even from now, that based on the results included in the figure (and, the PM definition - "PM should have at least 2 branches") for mechanisms with (2DOF) seems to be that this class doesn't have a representative. This is not so true(or only, partially), probably because of the lack of graphical representation. If we think at a planar motor(slider)-with 2 dof, its kinematic(mechanical) scheme would be seen, for instance, as a combination of two (serial) orthogonal Prismatic(P) joints. But, in fact, the best (true?) representation could be as in Fig. 1, by using 4 pairs (Prismatic, see Actuation). In the same time it can be seen as a (fully) 2 dof (actuated) planar mechanism with a single element (and, 2DOF actuator), and we should introduce it in the class. True, or not, we adopted it as a full member with all the rights and treated in accordance. And, moreover this will be exploited further in the benefit of the modularity. (It will be as a standard module inside of the each of the individual next superior structures (with 4,6 DOF) from the family, and the class. (And by itself as, the simplest structure for a positioning unit.) Between the structures some are new members and others were the basis of the architectures already mentioned previously, as potentially ones ${ }^{11,13}$.

Table 1. 2DOF PM family actuated from the base (structural systematization\&development)

\begin{tabular}{|c|c|c|c|c|c|c|c|c|c|c|}
\hline \multirow{2}{*}{$\begin{array}{l}\mathrm{D} \\
\mathrm{O} \\
\mathrm{F}\end{array}$} & \multirow{2}{*}{$\begin{array}{c}\text { Structure } \\
\text { DOF-k[KC] }\end{array}$} & $\mathrm{KC}$ & \multirow[t]{2}{*}{$\mathrm{k}$} & \multirow[t]{2}{*}{$\mathrm{n}$} & $\mathrm{N}$ & $\mathrm{c}_{5}$ & $\mathrm{c}_{4}$ & $c_{3}$ & ${ }^{* * *} \mathrm{c}_{2}$ & $\mathrm{C}$ \\
\hline & & $(\text { I })^{*}$ II III IV & & & $\mathrm{k} \Sigma \mathrm{n}+\mathrm{mP}$ & $\mathrm{P}, \mathrm{R}$ & $\begin{array}{l}\mathrm{U}, \mathrm{C}, \\
(\mathrm{PI})^{* *}\end{array}$ & $\mathrm{~S}$ & $\mathrm{~S}_{\mathrm{c}}, \mathrm{C}_{\mathrm{s}}$ & $\mathrm{k} \sum \mathrm{c}_{\mathrm{i}}$ \\
\hline \multirow[t]{2}{*}{2} & $2-2(1) 1$ & (1) 1 & 2 & 1 & 2 & 2 & & - & - & 4 \\
\hline & $2(2)^{* * *}$ & (2) & - & - & 1 & - & (1) & - & & 1 \\
\hline \multirow[t]{3}{*}{4} & $4-2[(2) 111]$ & (2) 111 & 2 & 3 & 7 & 3 & (1) & - & - & 8 \\
\hline & $4-2[(2) 12]$ & (2) 12 & & 2 & 5 & 1 & $(1)+1$ & - & - & 6 \\
\hline & $4-2[(2) 31]$ & (2) 31 & & & & 1 & (1) & 1 & 1 & \\
\hline \multirow[t]{7}{*}{6} & $6-3[(2) 1111]$ & (2) 1111 & 3 & 6 & 19 & 4 & (1) & - & - & 15 \\
\hline & $6-3[(2) 112]$ & (2) 112 & & 3 & 10 & 2 & (1) & - & - & 12 \\
\hline & $6-3[(2) 121]$ & (2) 121 & & & & & $(1)+1$ & - & - & \\
\hline & $6-3[(2) 211]$ & (2) 211 & & & & & $(1)+1$ & - & - & \\
\hline & $6-3[(2) 13]$ & (2) 13 & & 2 & 7 & 1 & (1) & 1 & - & 9 \\
\hline & $6-3[(2) 31]$ & (2) 31 & & & & & (1) & & - & \\
\hline & $6-3[(2) 4]$ & (2) 4 & & 1 & 4 & - & (1) & - & 1 & 6 \\
\hline
\end{tabular}

* Actuated, ** Proposal, *** ex. Sphere-Curve, Curve-Sphere

The members have a minimum number of kinematics chains, the number of joints and links. And, as a direct result, the direct and inverse (used in) kinematics will be more friendly (calibration, control,..), and the design and 
manufacturing conduct towards the low cost manipulator. Between the specific individual members (families) features- number of links(n) and joints $\left(c_{i}\right)$, or their standard type $(P, R, U, \ldots)$ in a chain $(k)$, or totally in the mechanism $(\mathrm{N})$ and $(\mathrm{C})$, the class exhibits a common and general feature-all actuators are at the base, and moreover, each members are in-parallel actuated, by a combination of one(1)), two(2) or three(3) from 2 dof actuation, as previously discussed.

Modularity. Generally, a Multi DOF PM (redundant, hybrid,...) is by its intrinsic structure a system susceptible to be designed in a modular way. This aspect is more evident if the PMs are with a symmetrical structure, as our selected structures are. The role and functional principle will be explained for every selected module.

Actuation Module (aM). There are several possibilities to introduce the motion in a planar or spatial parallel mechanism. In any cases, from the base or near by, we have clearly some advantages comparing with other more exotic, and as a result, less exploited solutions.

By the previous systematic structural systematization, the simplest structure of the class (with 2dof) could act as an standard actuator of the entire family(and, class), and by this, designed in accordance. (See the latter design issues for 2DOF module(Pu)). From kinematics point of view, and based on the scheme included in Fig.1/Tab.1, a parallel mechanism in this case should have at least two(2) kinematics chain KCs (from the definition) to be indeed with a parallel structure. We intend to use an in-parallel actuation device for actuators of all our PM structures selected as candidates.

As one of the fundamental design principles was to use the linear technology, not only to be sure that an adequate (high) precision and speed is assured, but to pass/encompass with the other requirement related with the work over appreciable long orthogonal distances (table top), a PrismaticPrismatic (PP) in-parallel (P-P) scheme was chosen, as a mechanism for input motion (Fig.2). It has four pair (P) and [2-2(P)P)] structure, to produce a planar actuated output motion at the end-effector (mobile platform).
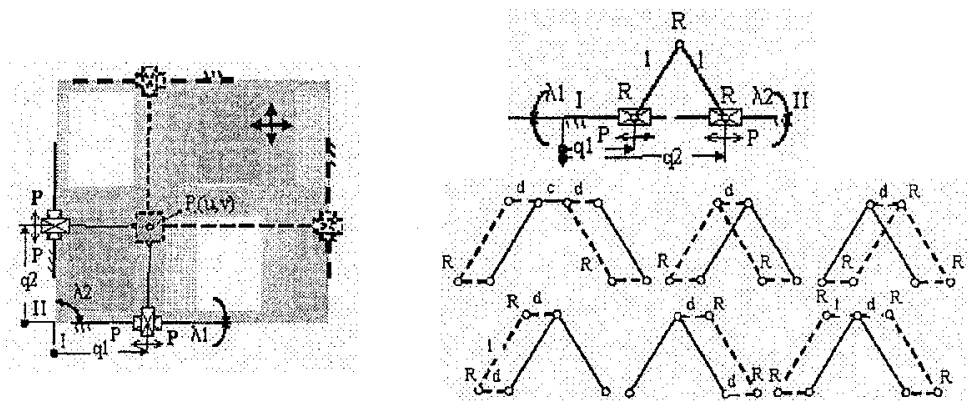

Figure 2. PM Actuation module(aM) and Transfer of Motion(TOfM) modules (kinematics) 
Transfer of Motion (TofM). Scissor mechanisms was from a long time used as a fast and simple 2 dof mechanism to transform a linear input motion (from one axis) to in plane(output) one, but more recently, an increased interest is seen with the development of machines with parallel kinematics(PKM) or, the auxiliary devices in (precision) assembly ${ }^{17,18}$

A considerable amount of study was allocated to 4-bars mechanism in the past, but an important theoretical and practical work has been done in the connection with more recently, parallel mechanisms developments. Four bar linkages group are closed loop mechanisms with a single dof input and the characteristic element can perform an output constrained motion in a plan, but between them, the particular case- parallelogram ( $\mathrm{Pa}$ ) was the most widely used as generator of motion in translations, from several years ago. Indeed, the (output) motion of the last element is the same with that of a body in planar translation. This can be from the real use in generating ample motions, as in our case, or for a variety of others purposes, clearly shown ${ }^{18}$.

The concept of mathematical Group Theory was used even from the beginning for the (analysis) and synthesis of PMs structures, and by this to find new structures (STAR). As was suggested there ${ }^{19}$, for 3 dof spatial translations, the parallelograms $(\mathrm{Pa})$ can be used to produce the expected results. DELTA use them as main principle and taken the advantages. Other researchers (re)discovered it within its planar configuration ${ }^{20}$.

In our design, we used these two generators of motions transformers scissor mechanisms(Sc) and parallelograms $(\mathrm{Pa})$, to increase the speed and stiffness for our class of the PS, and to have by this, ample motions capabilities inside of some new PM structures. Their fundamental principle applied in our case, as a combination of them are used as is shown in Fig.3 to develop new PSs architectures, and/or to improve the features of others. This concept can be extended with an adequate design at all the structures which have at least one rotation (R) pairs in their structure.

Mobile Platform (mP) module is based mainly on the end-effector component, which holds the necessary attachments - devices, in doing the scheduled assembly operations in the desktop cell with the required parameters. The shape is in accordance with these operations and its interactions. It could be as a tray for transfer/transportation, probe for control, grasping for manipulation, tools for small adjustments, screwing, etc and designed in accordance with the specific parameters.

\section{$2.2 \quad$ Positioning units $(\mathrm{Pu})$}

The modular concept of design PS as a unique PM structure was previously described. It consist from three modules: 1) actuation (aM), 2) Transfer of Motion (TofM) and mobile Platform (mP). This concept can be 
extended behind, only to be applied, at an individual structure. It can be a (general) concept for the entire family or, class, which (virtually) includes other members (serial connected, redundant, hybrids). This would be sure a serious advantage in the design and manufacturing costs of multi-agent, multipurpose PSs.

2DOF Pu. Based on kinematics of in-parallel actuation module (Fig.2), an independent unit with built-in functions-proper force and guidance can be designed. This integrated multi-degree-of freedom element (platform) as a direct drives unit -planar motors(Fig.3), is moving simultaneously through forces coming from a magnetic field, in two orthogonal axis $(X-Y)$, instead of two-one axis (electro) mechanical modules. They are proved to be a good choice for planar high speed precise manipulations/actuations- no friction and no wear, heat or dust, as a result of a air bearing suspension, being suitable for clean room environments. On the other hand, the concentration of the actuated degrees of freedoms in one unit has other advantages compared with more classical ones, by using selectively only one simple dof. Linear motion technology(direct-drive) now is evolving, and their advantages converted in compact, precise and fast planar modules. In our design ${ }^{21}$, a slider $(80 \times 80 \times 28 \mathrm{~mm})$ which, hold the coils, is moving suspended by the balance between the attracting forces and an air-slide bearing pushing force and moves on a base (desktop table) - platen $(760 \times 700 \times 15 \mathrm{~mm}$,) as a interaction of the coils with the magnetic (steel) environment based on stepper motor open loop principle (pitch-2 $\mathrm{mm}$ ) and. By the combinations (in a standard or, variable way- with the auxiliary contact or, not) this standard positioning unit(s) can be configured forming simple or complex planar shapes, or for the members of the class-Positioning units(Pu) with 4, 6 or more DOF caring materials, tool, components, finished products,...

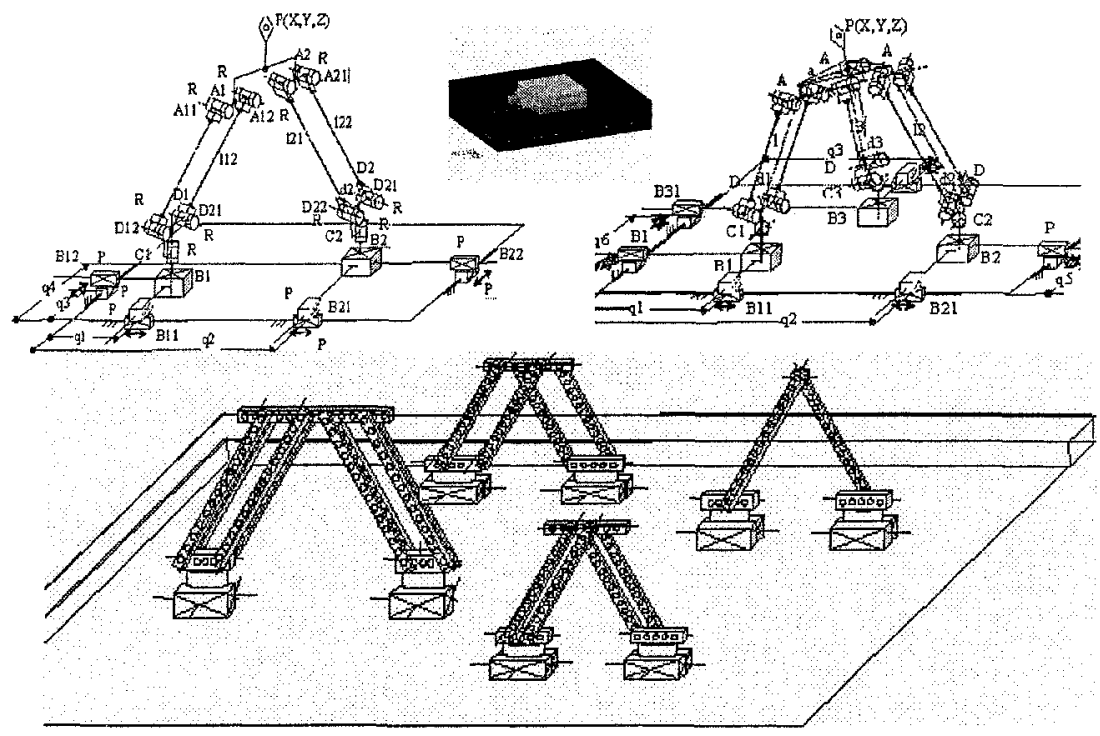


Fig.3. MDOF Pu -2, 4 and 6 DOF, kinematics: 2-(Pl), 4-2[(Pl)RPa and 6-3[(Pl)SPa] \& design

$4 D O F P u$. Based on the previous four structures included in Tab.1/ Fig.1 having 4DOF and a least one (1) dof pairs on a level, we want to draw the attention on 4-2[(2)111] structure. Taken in to account the possibilities to be designed as a combination of only simple common pairs ( $\mathrm{P}$ and/or $\mathrm{R}$ ) and their reciprocal spatial axis disposal $\left(0^{\circ}\right.$ and $/$ or $\left.90^{\circ}\right)$ one of the architectures could have the kinematics as the Fig.3, the kinematics scheme shows.

The architecture of the mechanism is based on the previous kinematics design principles for the actuation/motion transfer mechanisms, and an additional two $\mathrm{R}$ pairs, with the axes perpendicular on the actuation plane. Between the $\mathrm{mP}$ and these pairs $(\mathrm{R})$ two (for, symmetry only) planar vertical $\mathrm{Pa}$ or (RRRR) is moving. The result is that the end-effector (a rod) can perform, all three translations $(\mathrm{X}, \mathrm{Y}, \mathrm{Z}$ ) and one rotation (around vertical axis $(Z)$ ). As in most of the PM structures, every motion is generated and resulted from the cooperative actions(motions) of the actuation (sub) systems - the mechanisms has a high index of efficient use of actuators. With other words, an increased force(and/or torque) capabilities is seeing for the mechanics of motion in $\mathrm{x}$-axis, $\mathrm{y}$ - axis and $\mathrm{z}$-axis (including the rotation).

Depending on the mechanisms constructive parameters (link and base radius), an additional higher stiffness can be seen in the axial axis ( $z$ ) and longitudinal axis $(x)$ of the mechanisms, which can reduces the deflection and provides a higher-level natural frequency.

$6 D O F P u$. Based on the 6-3[(2)31] structure from Tab.1, a kinematics proposal for a mechanism with full DOF motion, 6-3(Pl)SPa, by using the same $(\mathrm{Pa})$ principles(s) is proposed, too.(Note, symmetrical counter parts, are to be taken into account, also: 4-2[(Pl)PaR and 6-3[(Pl)PaS], respectively.)

\section{CONCLUSIONS}

A challenging tasks today, in the precision manipulation, is to design flexible systems, for process like cell assembly, and with an increased accuracy for large moving volumes with micrometer accuracy. A selected class of PM was developed by using advanced actuation and high efficient motion transformers (mechanisms) in their kinematics. Several representative members, designed on the modular concept of 2 dof in-parallel actuation, and for a practical implementation as positioning/manipulation precision system in a highly environment controlled conditions(multi-agents, multi-assembly, multi-cells) have some specific features. An increased 
potential of use in term of stiffness and the capabilities to perform multi degrees of freedom motions tasks with accuracy required in some precision assembly operations (transport, manipulation, screwing, ...) from other fields of applications, like (microfactory, micromachining, microscope manipulations,...) can be further expected. For the experimental purpose, the system was successfully tested in an Attentive Work Bench (AWB) solution ${ }^{4}$ - component of an assembly cell, proving the kinematic and design concept. This work is indeed in progress, because we intend to study the system from the kinematic and dynamic point of view, and to identify more precise the members' potential, by using a systematic analytical approach and the chance to use an automatic computational algorithm for their analysis and simulation-ROBOTRAN ${ }^{22}$ (Multi Body Dynamics symbolic software).

\section{REFERENCES}

1. CPT , 2002, "Int. Conf. on New Technological Innovation for Positioning" Proc. of $1^{\text {st }}$ KOREAN-JAPAN Conf. on Positioning Technology, Daejon, Korea.

2. V. Scheinman, 1994, Robot World: a multiple robot vision guided assembly system, Rob. Res, Proc. $4^{\text {th }}$ Int. Symp. MIT Press, pp. 23-27.

3. A. E Quaid., and Hollis R., 1996, Cooperative 2-DOF Robots for Precision Assembly, Proc. Int. Conf. Rob. Aut., Mineapolis, US, pp.

4. H. Suzuki et all, 2003, An overview of Attentive Work Bench(AWB), Proc. Symp. Real World Information Systems, 21 Cent. COE, Tokyo, Japan, pp. 65-66.

5. J.P. Merlet, 2000,"Parallel Robots, KLUWER Academic Publisher, ISBN 0-7923-630

6. H. Mac Callion, and D.T. Pham, 1979, The analysis of a six degree of freedom work station for mechanized assembly, Proc. $5^{\text {th }}$ World Cong. Th. Mach.\&Mech., Montreal.

9. D. Stewart, 1965,"A platf. with 6 deg. of freedom",Proc.Inst.Mch.eng.,180,15,pp.371386

10. R. Clavel,1988,DELTA, a fast Robot with Parallel Geometry, Proc.Int.SymRob.,pp.99109.

11. F. Tahmasebi et all., 1994, Six degrees of freedom "minimanipulator" with three inextensible limbs, US Pat. 5279176.

12. D. Kohli et all., 1998, Manipulator Config. Based on Rotary Linear (R-L) Actuators and their Direct and Inverse Kinematics, ASME J. Mech.Transm. Aut. 110, 4, pp.397-404.

13. R. Ben Horin, 1994, A Six Degrees of Freedom Parallel Robot with Three Planarly Actuated Links, Ms.Th., Technion, Israel.

14. G. Olea, 1999, Contribution to the Kinematics, Dynamics and CAD of "A" Type Spatial Parallel Mechanisms, in Three Points Guided, Ph.D,Th., Tech. Univ. Cluj, Romania.

15. G. Olea, 2000, Parallel Mechanisms with Planar Actuators -The general constructive features, Tech. Rep.(internal use), Univ. Of Tokyo, Japan.

16. G. Olea, et all, 2001, Spatial Parallel Mechanism with 2DOF L-L Actuators, 32-nd Int. Symp. Rob., Seoul, Korea, pp.1129-1134.

17. G. Olea, et all., 2003, Development o Parallel Positioning Systems for Precise Micro/Mini Applications, Proc. IPAS' 2003, Bad Hofgastein, Austria, pp.95-101

18. SFB 562, Robotersysteme fur Hanhabung und Montage, www.tu-braunschweig.de/sfb562 
19. M. Herve, 1991, Structural Synthesis of "Parallel" Robots Generating Spatial Translations, Fifth Int. Conf. On Ad. Rob. ICAR91, June 19-22, Pisa, Italy, pp.808-813

20. X-J. Liu and J. Wang, 2003, Some New Parallel Mechanisms Containing the Planar Four-

Bar Parallelogram, Int. J, Rob. Res. Vol. 22, pp. 717-732.

21. X. Chen, et all, 2004, Dev. of Planar Linear Motors System, Proc. of the JSPE Annual Meeting, September 15-17, , Fukui, Shimane, JAPAN, B18(CD).

22. ROBOTRAN, http://www.prm.ucl.ac.be/robotran/ 